\title{
Benignes fibröses Histiozytom des Knochens: MR-Diagnostik
}

Fibröse histiozytäre Läsionen des Knochens umfassen neben dem nicht-ossifizierenden Fibrom und dem Riesenzelltumor auch das seltene benigne fibröse Histiozytom. Da dieser Tumor histologisch vom nicht ossifizierenden Fibrom kaum zu unterscheiden ist, kommt der Bildgebung neben Anamnese und klinischer Symptomatik bei der Diagnosestellung große Bedeutung zu (Jundt G, Orthopäde 1995; 24: 2). Bisher sind in der Literatur nur wenige Fälle beschrieben (Dahlin DC, Bone Tumors 1986; 522). Insbesondere zur MR-Diagnostik des benignen fibrösen Histiozytoms liegen bisher kaum Daten vor.

\section{Fallbericht}

Ein 20-jähriger Patient stellte sich mit seit 9 Monaten bestehenden Schmerzen im Bereich des linken Kniegelenkes vor. Die körperliche Untersuchung war unauffällig. Zur weiteren Abklärung der Schmerzsymptomatik wurden Röntgenaufnahmen des linken Kniegelenkes angefertigt. Dabei zeigte sich ein $6 \times 2 \times 2 \mathrm{~cm}$ messender, exzentrisch wachsender, zystisch-septierter Tumor, der mit seinem Hauptteil in der proximalen Fibuladiaphyse mit Übergriff auf die Metaphyse liegt (Abb.1). Dieser war auf einer 11 Jahre zuvor angefertigten Röntgenaufnahme des Kniegelenkes nicht nachweisbar. Im Rahmen der präoperativen Planung wurde eine Kernspintomographie durchgeführt. Dabei wies die Läsion ein inhomogenes Signalmuster auf. Die Signalintensität war sowohl auf den frequenzselektiv fettsupprimierten als auch auf den $\mathrm{T}_{1}$ - und $\mathrm{T}_{2}$-gewichteten Aufnahmen hypointens (Abb. 2). Nach Gabe von Gd-DTPA wies die Läsion einen ungleichmäßig verteilten Signalanstieg auf. Es zeigten sich einzelne sowohl in den $\mathrm{T}_{1}$ - als auch den $\mathrm{T}_{2}$-gewichteten Bildern signalfreie Areale ohne Kontrastmittelaufnahme. Ein umschriebener Bezirk blieb sowohl auf den $\mathrm{T}_{1}$ - als auch auf den $\mathrm{T}_{2}$-gewichteten Bildern signalreicher als der umliegende Tumor. Die Kortikalis war medialseitig hochgradig ausgedünnt, jedoch nicht durchbrochen.

Der Tumor wurde kürettiert und der Operationsdefekt mit autologer Spon- giosa aufgefüllt. Der operative und postoperative Verlauf waren komplikationslos. Der Patient war kurze Zeit nach Operation völlig beschwerdefrei. Das histologische Präparat zeigte eine Fibrosierung des Markraumes mit einzelnen Einblutungen. Weiterhin ließen sich Anhäufungen von Bindegewebe umgebener Schaumzellen und Areale mit einzelnen Riesenzellen nachweisen. Zellkernatypien lagen nicht vor. Es wurde die Diagnose eines benignen fibrösen Histiozytoms gestellt.

Während einer bisher 4-jährigen Nachbeobachtungsperiode ergaben sich keine Hinweise für ein Rezidiv.

\section{Diskussion}

Beim benignen fibrösen Histiozytom handelt es sich um einen seltenen benignen Knochentumor über den bisher nur vereinzelt berichtet wurde. Das histologische Bild ähnelt dem des nicht ossifizierenden Fibroms. Aufgrund dieses Umstandes ist eine sichere histopathologische Zuordnung in Abgrenzung vom nicht ossifizierenden Fibrom und Riesenzelltumor schwierig. Beide Tumorentitäten zeigen histologisch fibroblastische Spindelzellen und histiozytäre Anteile, mit zwischengelagerten Arealen von lipidhaltigen Makrophagen. Riesenzellen können vorkommen. Die Zellkernmorphologie ihrerseits ist unauffällig. Die Ätiologie ist bisher nicht abschließend geklärt. Eine ätiologische Verwandtschaft zum nicht ossifizierenden Fibrom, zum Riesenzelltumor und zum malignen fibrösen Histiozytom wird diskutiert (Hamada et al., Skeletal Radiol 1996; 25: 25).

Während das nicht ossifizierende Fibrom typischerweise im Kindes- und Jugendalter auftritt, handelt es sich beim benignen fibrösen Histiozytom um einen Tumor des jungen Erwachsenen, der nur äußerst selten im Jugendalter zu finden ist. Das benigne fibröse Histiozytom findet sich im Gegensatz zum metaphysär in den langen Röhrenknochen gelegenen nicht ossifizierenden Fibrom zumeist in der Epiphyse oder Diaphyse der Röhrenknochen. Auch in anderen Skelettabschnitten, insbesondere Be-

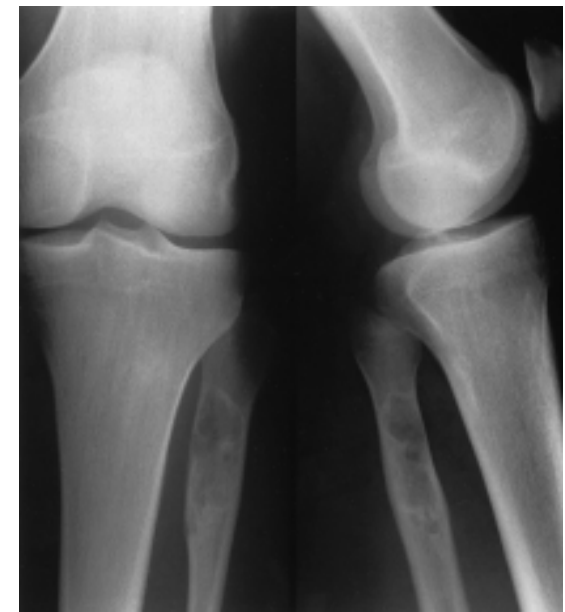

Abb. 1 Die Röntgenaufnahme des Kniegelenkes zeigt einen exzentrisch wachsenden, osteolytischen Tumor der proximalen Fibula. Neben einer Septierung weist die Raumforderung eine Randsklerose auf.

cken, Rippen und Wirbelkörper, wurde das benigne fibröse Histiozytom beschrieben. Ein weiteres Unterscheidungsmerkmal ist die klinische Symptomatik. Das benigne fibröse Histiozytom äußert sich im Regelfall durch lokale Schmerzen, während das nicht ossifizierende Fibrom zumeist erst nach einer pathologischen Fraktur Beschwerden bereitet. Im Gegensatz zu anderen fibrösen histiozytären Läsionen sind beim benignen fibrösen Histiozytom lokal aggressives Wachstum und wiederholte postoperative Rezidive beschrieben (Statz EM et al., Skeletal Radiol 1989; 18: 299).

Die konventionelle Röntgenaufnahme bei benignem fibrösen Histiozytom zeigt eine lytische, expansive Läsion, die ihrerseits Trabekulierungen aufweisen kann. Randsklerosen können vorkommen. Bisher wurde in der Literatur nur in 4 Fällen über den MR-tomographischen Befund bei einem benignen fibrösen Histiozytom des Knochens berichtet. Die MRT zeigt, wie beim häufigeren benignen fibrösen Histiozytom des Weichgewebes, in den $\mathrm{T}_{1}$-gewichteten Aufnahmen eine inhomogene, hypointense Signalintensität, die in weiten Anteilen isointens zum Muskelgewebe ist. Die von uns beschriebene Signalcharakteristik der $\mathrm{T}_{1}$-gewichteten Aufnahmen entspricht dem bisher in der Literatur beschriebenen Erscheinungsbild. Die $\mathrm{T}_{2}$-gewichteten Bilder betonen die Signalinhomogenität, wobei der Tumor geringfügig signalreicher erscheinen kann. Verein- 

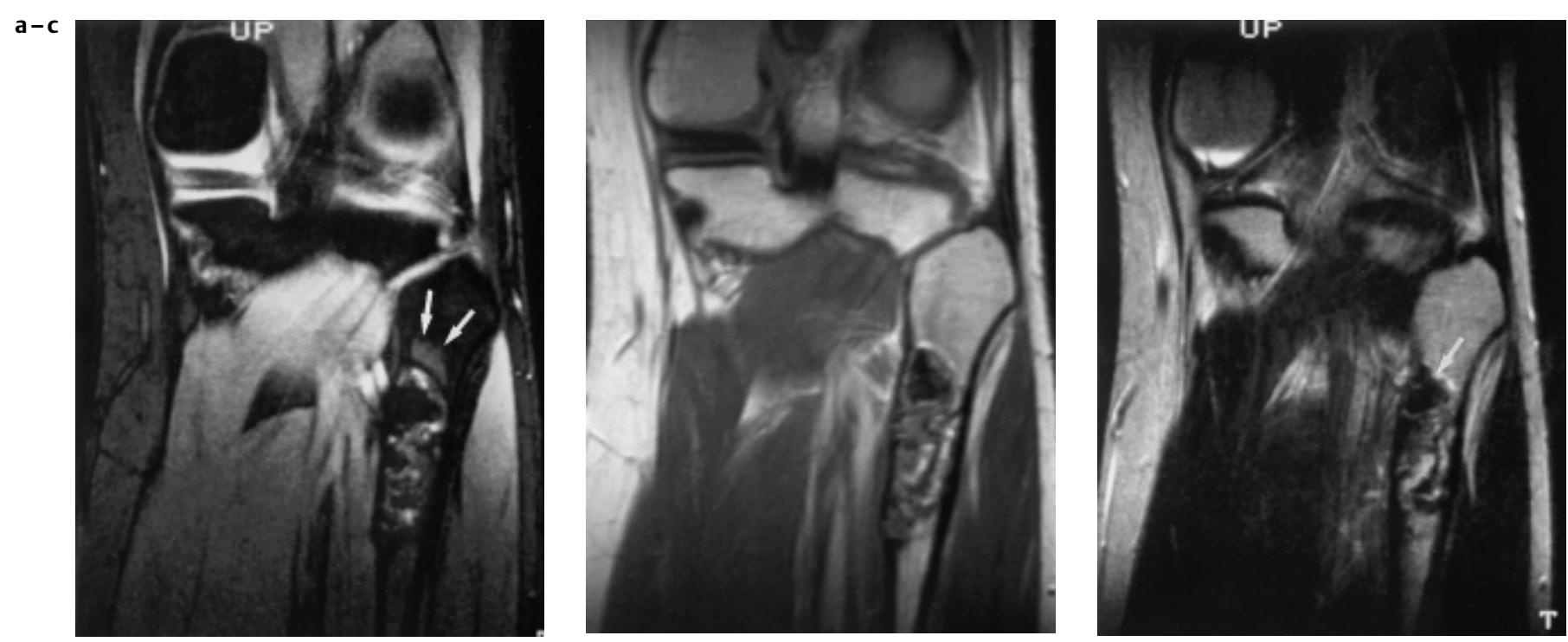

Abb. 2 (a) Die frequenzselektive Fettsuppression (chemical shift selective saturation (CHESS), $T_{R} 510 / T_{E} 12 /$ Flipwinkel $45^{\circ}$ ) zeigt eine signalinhomogene, hypointense Raumforderung mit im kranialen Anteil nachweisbarem perifokalen Ödem (Pfeile). (b) Auf der $\mathrm{T}_{1^{-}}$ gewichteten Aufnahme $\left(T_{R} 600 / T_{E} 15\right)$ stellt sich der zentrale Tumoranteil isointens zum Muskelgewebe dar. Die weitere Signalverteilung ist inhomogen mit signalfreien Abschnitten und einzelnen signalreichen Anteilen, die histologisch mit Ansammlungen von lipidreichen Schaumzellen übereinstimmen. (c) Auf der $T_{2}$-gewichteten Aufnahme $\left(T_{R} 2300 /\right.$ $T_{E}$ 90) bleiben die in den $T_{1}$-gewichteten Aufnahmen als signalfrei beschriebenen Abschnitte hypointens (Pfeil), histologisch Hämosiderinablagerungen entsprechend. zelt wurde bei $\mathrm{T}_{2}$-gewichteten Aufnahmen über eine deutlich erhöhte Signalintensität berichtet (Exner GU et al., Z Orthop 1990; 128: 308). Zentral zeigt sich in unserem Fall ein zu Fett isointenses Areal. Dies entspricht histologisch einer Anhäufung lipidhaltiger Makrophagen, sogenannter Schaumzellen. Eine Diskrepanz liegt zu dem von Hamada et al. 1996 berichteten Erscheinungsbild nach Fettsuppression vor. Während dort nach einer STIR-Sequenz über ein hyperintenses Signalverhalten berichtet wird, kommt es bei dem hier berichteten Tumor nach frequenzselektiver Fettsuppression zu einem hypointensen Signalverhalten. Dies ist in unseren Augen Ausdruck der bei dem hier berichteten Fall zahlreich vorhandenen lipidreichen Schaumzellen. Über das Kontrastmittelverhalten des benignen fibrösen Histiozytoms ist bisher nach unserem Wissen nicht berichtet worden. Bei dem hier beschriebenen Tumor kommt es nach Kontrastmittelgabe $\mathrm{zu}$ einem mäßigen ungleichmäßig verteilten Signalintensitätsanstieg. Der Tumor weist weiterhin in der MRT Areale auf, die sich sowohl auf den $\mathrm{T}_{1}$ - als auch $\mathrm{T}_{2}$-gewichteten Aufnahmen hypointens darstellen und keinerlei Kontrastmittel aufnehmen. Diese entsprechen den histologisch beschriebenen Einblutungen. Ein perifokales Ödem zeigt sich kranial des Tumors und unterstreicht den als aggressiv beschriebenen
Charakter der Läsion. Ein extraossärer Tumoranteil ist nicht nachweisbar.

Zusammenfassend betrachtet ermöglicht auch die MRT keine eindeutige Differenzierung des benignen fibrösen Histiozytoms vom nicht ossifizierenden Fibrom oder Riesenzelltumor. Lediglich das perifokale Ödem liefert einen Hinweis auf den im Vergleich zum nicht ossifizierenden Fibrom aggressiveren Charakter des benignen fibrösen Histiozytoms. Klinische Angaben zur Be- schwerdesymptomatik und konventionelle Röntgenaufnahmen zur Lokalisationsdiagnostik bleiben die notwendige Basisdiagnostik. Die MRT sollte ergänzend bei konventionell radiologisch aggressivem Erscheinungsbild und falls notwendig im Rahmen der Operationsplanung zur Erkennung eines eventuellen extraossären Tumoranteiles herangezogen werden.

A. H. Mahnken, S. Biesterfeld, J. E. Wildberger, Aachen 